\title{
Amelioration of Persistent, Non-Ketotic Hyperglycemia-Induced Hemichorea by Repetitive Transcranial Magnetic Stimulation
}

\author{
Yumiko Kaseda ${ }^{a}$ Takemori Yamawaki $^{\mathrm{b}}$ Junko Ikeda ${ }^{\mathrm{a}} \quad$ Miwa Hayata $^{\mathrm{a}}$ \\ Eisuke Dohi $^{\mathrm{b}} \quad$ Tomohiko Ohshita $^{\mathrm{c}}$ Kazuhide Ochi $^{\mathrm{b}}$ Eiichi Nomura ${ }^{\mathrm{c}}$ \\ Masayasu Matsumotob \\ ${ }^{a}$ Department of Neurology, Hiroshima City General Rehabilitation Center, ${ }^{b}$ Division of \\ Integrated Medical Science Programs for Biomedical Research, Department of Clinical \\ Neuroscience and Therapeutics, Hiroshima University Graduate School of Biomedical \\ Sciences, and 'Department of Neurology, Suiseikai Kajikawa Hospital, Hiroshima, Japan
}

\section{Key Words}

Hemichorea - Hyperglycemia - Repetitive transcranial magnetic stimulation - Basal ganglia MRI $\cdot$ Rehabilitation

\begin{abstract}
Diabetic hemichorea-hemiballism with non-ketotic hyperglycemia is usually a benign syndrome. Here, we report a 78-year-old woman with persistent hemichorea (HC) for longer than 1 year with a recurrence after rapid correction of hyperglycemia. Following the disappearance of the characteristic T1 hyperintensity at 3 months after onset, an MRI demonstrated $\mathrm{T}^{*}$ hypointensity and atrophic changes in the contralateral striatum, suggesting irreversible neuronal loss and some vascular proliferation. The electrophysiological examination using transcranial magnetic stimulation revealed significantly shorter cortical silent periods (CSPs) on the contralateral primary motor cortex (M1), possibly in relation to cortical hyperexcitability. We have applied 10 daily sessions of low-frequency repetitive transcranial magnetic stimulation (rTMS) over the contralateral M1 to reduce the hyperexcitability. The $\mathrm{HC}$ was suppressed during and for several minutes after rTMS with prolongation of CSPs. After rehabilitation therapy, the patient was able to walk independently with a walker. We suggest that the combination of low-frequency rTMS and rehabilitation therapy may be a possible choice in medically refractory involuntary movements.
\end{abstract}




\section{Introduction}

Diabetic hemichorea-hemiballism (HC-HB) with non-ketotic hyperglycemia, accompanied by contralateral striatal hyperintensity on T1-weighted MRI, has been reported mainly in elderly Asian women [1, 2]. The occurrence of HC-HB has been explained by motor cortical hyperexcitability induced by disinhibition of the thalamocortical pathway [3], and HC-HB usually resolves following blood sugar correction [1-3].

\section{Case Report}

A 78-year-old woman developed an acute onset of left-sided hemichorea (HC) following overeating and weight loss of approximately $10 \mathrm{~kg}$ over several months. On admission to the hospital, she was fully oriented, and the neurological examination was unremarkable except for left-sided HC. The blood sugar was $567 \mathrm{mg} / \mathrm{dl}$ with HbA1c of $13.9 \%$. The diagnosis of diabetic $\mathrm{HC}$ was confirmed by a high-intensity signal in the right striatum on T1-weighted MRI. Insulin therapy decreased HbA1c to the normal range within 1 month, and the HC nearly resolved, but relapsed 1 week later and progressed to severe $\mathrm{HC}$-HB persistent to haloperidol and clonazepam. The patient was transferred to the rehabilitation hospital 5 months after the onset of the left HC. She could not eat, take care of herself, or keep standing without assistance because of the jerky movements. Physical and occupational therapy was carried out for $2 \mathrm{~h}$ every day for 6 months after admission to the rehabilitation hospital.

A follow-up MRI examination showed a low-intensity signal on T2* images following the disappearance of T1 hypointensity at 3 months and atrophic changes at 8 months in the right striatum (fig. 1).

The routine electroencephalography and sensory-evoked potentials by median nerve stimulation were normal. The motor cortical excitability was examined by transcranial magnetic stimulation (TMS) using a double 7-cm figure-of-eight coil connected to a Magstim Super Rapid (Magstim, Whitland, Dyfed, UK). Motor-evoked potentials (MEPs) and cortical silent period (CSP), induced by magnetic stimulation to the primary motor cortex (M1), were recorded from the first dorsal interosseous (FDI). The sampling rate of the electromyogram was $10 \mathrm{kHz}$. The patient was comfortably seated in a reclining chair and was told to relax. The stimulating coil was placed over the motor cortex (6-7 cm lateral to the $\mathrm{Cz})$, and the location was adjusted so as to evoke the maximum MEP in the contralateral FDI. The optimal coil position was marked with a pen for the consistent placement of the coil. The resting motor threshold (rMT) was defined as the minimum stimulus intensity to induce a MEP of $\geq 50 \mu \mathrm{V}$ in at least 5 out of 10 consecutive trials, as previously described [4]. The CSP was examined under tonic maximal contraction of the FDI at a stimulus intensity of the rMT $\times$ 1.3. The latency and amplitude of MEPs were normal. The rMT on the right and left M1 stimulation was 70 and $65 \%$ of the maximum stimulator output, respectively, thus both being within normal range (mean \pm SD, $73 \pm 12 \%$ in the controls aged $\geq 65$ years old). The average duration of 5 CSP trials was $86 \pm 7$ and $176 \pm 8$ ms (mean \pm SD) on the right and left M1 stimulation, respectively. The significantly shorter duration of CSP on the right M1 stimulation (ANOVA; $F=18.273, \mathrm{p}<0.01$ ) may be related to hyperexcitability of the right motor cortex.

We planned to suppress the hyperexcitability by low-frequency repetitive TMS (rTMS) following 1 month of rehabilitation therapy and 6 months after the onset of the left HC. Written informed consent, approved by the Institutional Review Board of the Hiroshima City General Rehabilitation Center, was obtained from the patient and her family. 100 pulses of 
$0.2 \mathrm{~Hz}$ rTMS, with a stimulus intensity of $\mathrm{rMT} \times 0.9$, were applied over the right M1 daily for 10 days. Sham stimulation was performed on a separate day between day 5 and day 6 of the rTMS treatment. The left-sided HC was suppressed during and for several minutes after rTMS. The therapeutic effect was not observed on sham stimulation. CSPs on the right M1 stimulation were examined immediately before and after rTMS on days 1, 6, 8, and 10 of the rTMS treatment. In order to assess the immediate and long-term effects of rTMS, we compared CSPs recorded before and after rTMS treatment and among treatment day (time) by Fisher's two-way ANOVA (treatment $\times$ time) with repeated measure. The statistical analysis showed significant factor effects of treatment $(\mathrm{F}=21.7747, \mathrm{p}<0.01)$ and time $(\mathrm{F}=$ $8.7068, \mathrm{p}<0.01$ ). Overall, the duration of CSPs was significantly prolonged from $102 \pm 32$ to $145 \pm 40$ ms (mean \pm SD) $(p<0.01)$, but not by sham stimulation. CSPs recorded on day 10 were significantly longer than those recorded on days 1,6 , and 8 ( $p<0.01$ ) (fig. 2).

During the rTMS treatment period, the physical and occupational therapy was given after rTMS. After 6 months of rehabilitation, the HC gradually decreased so that the patient was able to eat by herself and walk with a walker, without any assistance.

\section{Discussion}

Diabetic HC-HB has been reported as a benign syndrome, with complete amelioration within 10 months in $76 \%$ of patients [1]. The striatal T1-hyperintense lesion, explained as petechial hemorrhage [5] or ischemia [1, 3], usually disappears after clinical recovery [1-3]. In some cases with persistent or recurrent HC-HB, gross fluctuations of blood glucose, including recurrent hypoglycemia [6] or rapid correction of hyperglycemia [1], have been reported (as in the present case). In the subacute-to-chronic phase, the progressive neuronal loss has been demonstrated as atrophy [7, 8] and as a cystic lesion [3], and the possibility of vascular abnormality has been suggested by gadolinium-enhanced striatal lesions on T1weighted images [9]. Recently, brain needle biopsy has revealed obliterative vasculopathy with prominent vascular proliferation, analogous to proliferative retinopathy [2]. Considering the recurrence after rapid correction of blood sugar and the increased striatal T2* hypointensity in the present case, the pathogenesis of striatal lesions in the chronic phase may be similar to that of diabetic retinopathy.

It has been reported that hyperglycemia shifts cerebral metabolism to an anaerobic pathway, and that striatal GABAergic neurons are particularly vulnerable to ischemia because GABA may be metabolized to succinic acid as an alternative source of energy [1]. The selective loss of striatal GABAergic neurons may be related to disinhibition of the thalamocortical pathway, resulting in the motor cortical hyperexcitability in diabetic HC [3]. It has been reported that CSP is mediated by GABA-B receptors [10]. The shorter CSPs on the affected side may be related to decreased inhibition mediated by GABAergic mechanisms. In writer's cramp, the cortical hyperexcitability has been demonstrated, and the subthreshold low-frequency rTMS suppressed dystonia for $3 \mathrm{~h}$ to days with prolongation of CSPs $[11,12]$. We have shown both immediate and the long-term changes in CSPs during the rTMS treatment. The long-term neurophysiologic changes may reflect the decreased cortical hyperexcitability with neuroplastic changes induced by rTMS [10]. Recently, it has been reported that 10 daily sessions of low-frequency rTMS, decreasing interhemispheric inhibition, promoted motor improvements for up to 3 months in chronic stroke patients and that greater outcomes were found in patients receiving rTMS before rehabilitation than in patients receiving rTMS after rehabilitation therapy [13]. rTMS may enhance use-dependent neural plasticity induced by rehabilitation therapy [13]. We suggest that the combination of 
rTMS and rehabilitation therapy may be a possible choice in the treatment of involuntary movement resistant to medication.

\section{Acknowledgements}

We deeply thank Hitoshi Sunahori, RPT, MS, Aiko Kiso, OTR, and Hiroyuki Moriuchi, RPT, MS, for their contribution in rehabilitation therapy.

\section{References}

-1 Oh SH, Lee KY, Im JH, Lee MS: Chorea associated with non-ketotic hyperglycemia and hyperintensity basal ganglia lesion on T1-weighted brain MRI study: a meta-analysis of 53 cases including four present cases. J Neurol Sci 2002;200:57-62.

2 Abe Y, Yamamoto T, Soeda T, Kumagai T, Tanno Y, Kubo J, Ishihara T, Katayama S: Diabetic striatal disease: clinical presentation, neuroimaging, and pathology. Intern Med 2009;48:1135-1141.

-3 Shan DE, Ho DMT, Chang C, Pan H-C, Teng MMH: Hemichorea-hemiballism: an explanation for MR signal changes. AJNR Am J Neuroradiol 1998;19:863-870.

-4 Ziemann U, Koc J, Reimers CD, Finkenstaedt M, Paulus W: Exploration of motor cortex excitability in a diabetic patient with hemiballism-hemichorea. Mov Disord 2000;15:1000-1005.

5 Chang MH, Chiang HT, Lai PH, Sy CG, Lee SS, Lo YY: Putaminal petechial haemorrhage as the cause of chorea: a neuroimaging study. J Neurol Neurosurg Psychiatry 1997;63:300-303.

6 Hefter H, Mayer P, Benecke R: Persistent chorea after recurrent hypoglycemia. Eur Neurol 1993;33:244247.

7 Iwata A, Koike F, Arasaki K, Tamaki M: Blood brain barrier destruction in hyperglycemic chorea in a patient with poorly controlled diabetes. J Neurol Sci 1999;163:90-93.

8 Yamawaki T, Isa K, Watanabe Y, Moriwaki H, Miyashita K, Nagatsuka K, Naritomi H: A long-term neuroimaging follow-up study in a case of hemichorea-hemiballism with non-ketotic hyperglycemia. Mov Disord 2000;15(suppl 3):231.

9 Battisti C, Forte F, Rubenni E, Dotti MT, Bartali A, Gennari P, Federico A, et al: Two cases of hemichoreahemiballism with nonketotic hyperglycemia: a new point of view. Neurol Sci 2009;30:179-183.

10 Hallett M: Transcranial magnetic stimulation: a primer. Neuron 2007;55:187-199.

-11 Siebner HR, Tormos JM, Ceballos-Baumann AO, Auer C, Catala MD, Conrad B, Pascual-Leone A: Lowfrequency repetitive transcranial magnetic stimulation of the motor cortex in writer's cramp. Neurology 1999;52:529-537.

12 Murase N, Rothwell JC, Kaji R, Urushihara R, Nakamura K, Murayama N, Igasaki T, Sakata-Igasaki M, et al: Subthreshold low-frequency repetitive transcranial magnetic stimulation over the premotor cortex modulates writer's cramp. Brain 2005;128:104-115.

13 Avenanti A, Coccia M, Ladavas E, Provinciali L, Ceravolo MG. Low-frequency rTMS promotes use-dependent motor plasticity in chronic stroke: a randomized trial. Neurology 2012;78:256-264. 


\section{Case Reports in Neurology}

\begin{tabular}{|c|c|}
\hline \multicolumn{2}{|c|}{ Case Rep Neurol 2013;5:68-73 } \\
\hline DOI: $10.1159 / 000350434$ & $\begin{array}{l}\text { (c) } 2013 \text { S. Karger AG, Base } \\
\text { www.karger.com/crn }\end{array}$ \\
\hline
\end{tabular}

Kaseda et al.: Amelioration of Persistent, Non-Ketotic Hyperglycemia-Induced

Hemichorea by Repetitive Transcranial Magnetic Stimulation

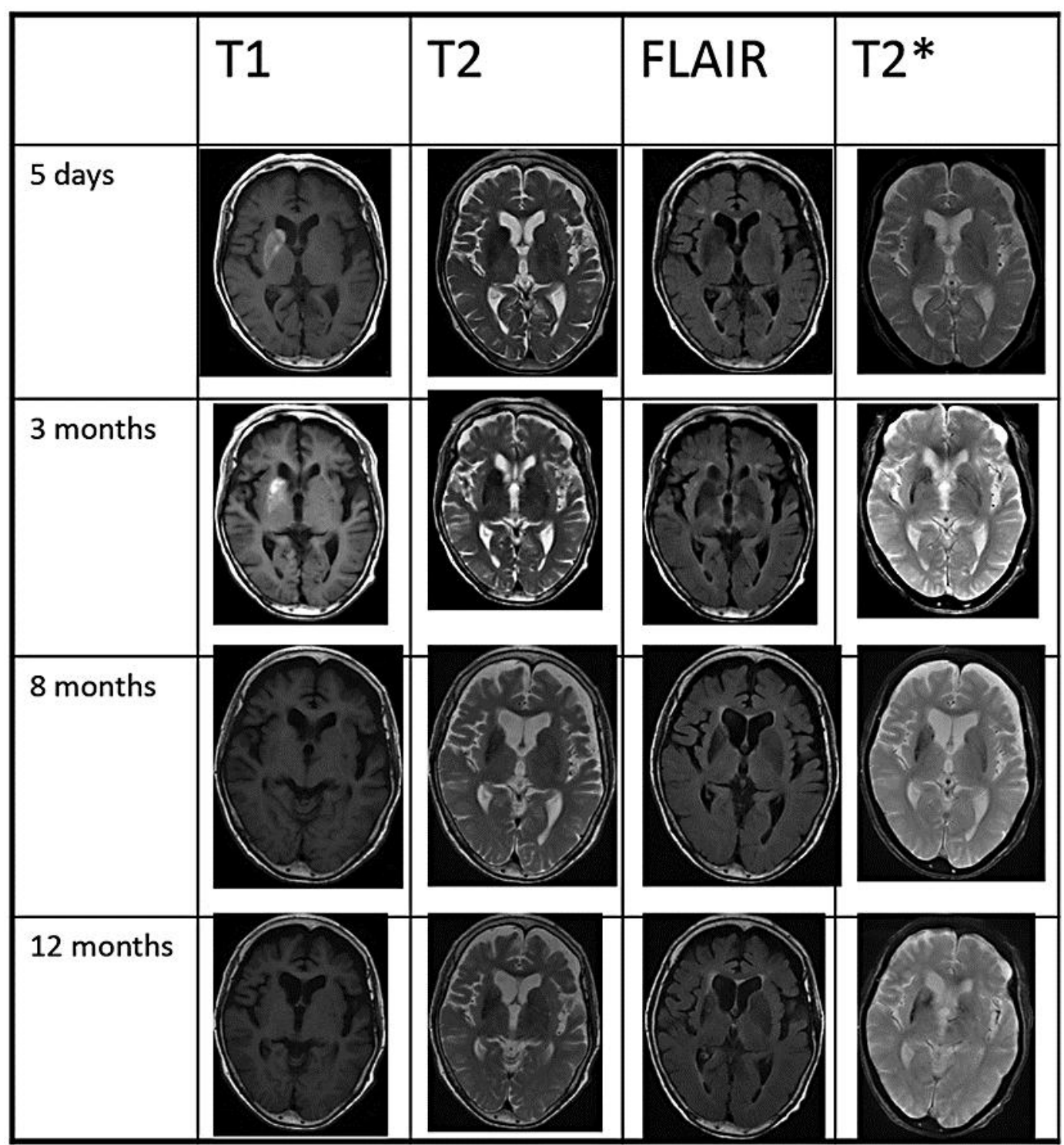

Fig. 1. MRI. T1-weighted images revealed hyperintensity in the entire right striatum (putamen, caudate head and globus pallidus) at day 5 and at 3 months. The right striatum was slightly hypointense in T2weighted and FLAIR images. T2*-weighted images demonstrated a low-intensity signal in the striatum predominantly on the right side at 3, 8 and 12 months. Atrophy of the right striatum was observed at 8 and 12 months. MRI was performed at the following facilities: day 5 (Suiseikai Kajikawa Hospital), 3 months (Hiroshima University School of Medicine), 8 and 12 months (Hiroshima General Rehabilitation Center). 


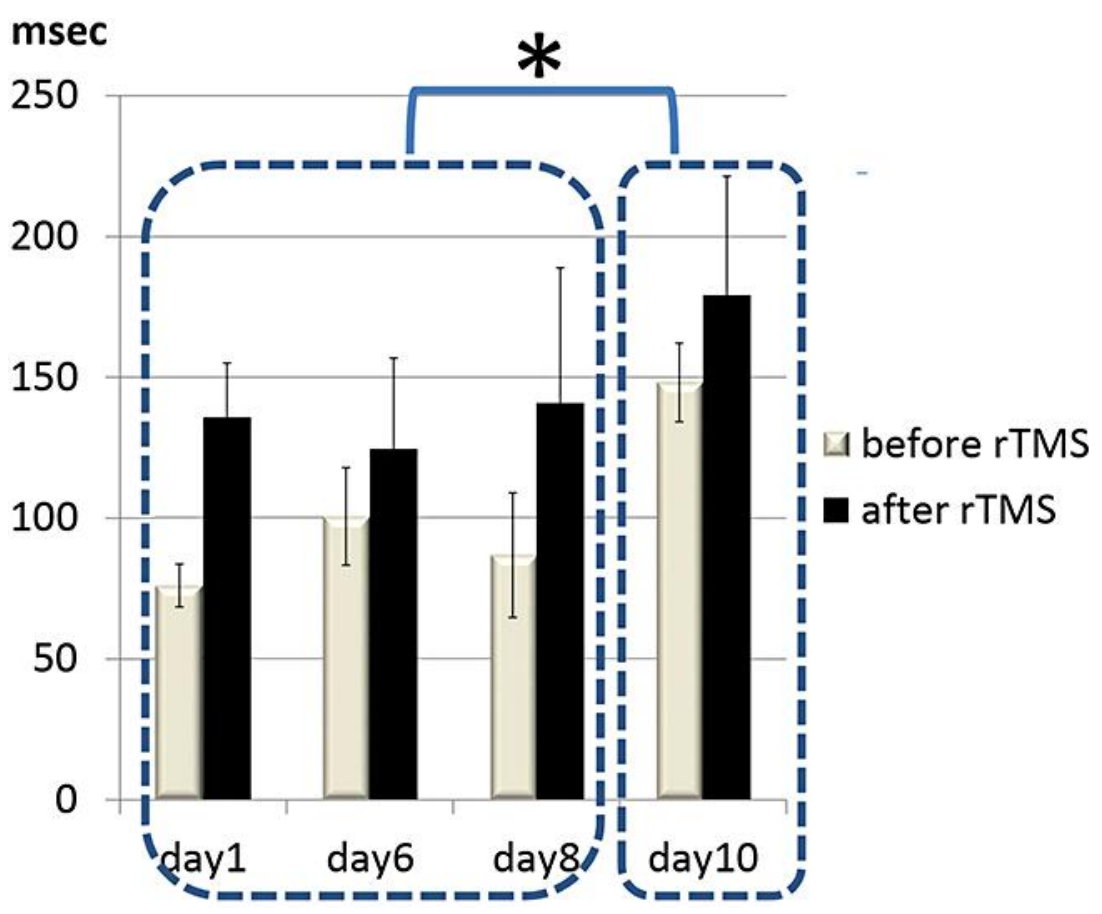

Fig. 2. Changes in CSPs during rTMS treatment. The CSPs on the right M1 stimulation were examined immediately before and after rTMS during rTMS treatment (days 1, 6, 8 and 10). The mean values \pm SD of 5 CSP trials are presented. CSPs were significantly prolonged by rTMS ( $<<0.01)$, and CSPs recorded on day 10 were significantly longer than those recorded on days 1,6 and $8(* p<0.01)$. 